\title{
The Laser Interferometer Gravitational Wave Observatory (LIGO) project
}

\author{
Stanley E Whitcomb \\ California Institute of Technology, 102-33, Pasadena, CA, 91125, USA
}

\begin{abstract}
The LIGO (Laser Interferometer Gravitational-wave Observatory) project $\dagger$ is designed to open a new field of science by detecting and studying the gravitational waves from astrophysical sources, including neutron stars, black holes, and possibly, supernovae and the big bang. LIGO will consist of two scientific facilities, each incorporating an L-shaped vacuum system with 4 -kilometers arms to house sensitive interferometers. A detector system consists of three interferometers, two at one site and one at the other. Each interferometer measures the motion of a set of test masses which are suspended from seismically isolated supports and free to move in response to gravitational waves. Correlations among the three interferometers will be used to eliminate local noise. LIGO is designed to support a sequence of detector systems of increasing sensitivity over the next twenty years or longer. In its initial configuration, it will have just one detector system. However, its design permits expansion to support three simultaneous detector systems. The project received funding in 1992 to begin design and construction. Sites for the two facilities (Hanford, Washington and Livingston, Lousiana) have been selected. Under the present schedule, the facilities will be completed by 1997 and initial observations will begin in 1998. Ultimately, the LIGO will be operated in coordination with interferometers in Europe and elsewhere, to form a worldwide gravitational wave observatory network. A more complete description of LIGO can be found in Abramovici et al [1].
\end{abstract}

\section{References}

[1] Abramovici A, Althouse W E, Drever R W P, Gürsel Y, Kawamura S, Raab F J, Shoemaker D, Sievers L, Spero R, Thorne K, Vogt R E, Weiss R, Whitcomb S and Zucker M 1992 Science 256 $325-33$

† This work is supported in part by NSF grant PHY-9210038. 